\title{
Connected Health Cities - Lancaster - the north west coast and beyond
}

\author{
Dr Jo Knight, Reader in Applied Data Science, Lancaster Medical School \& Data Science Institute \\ Mr Nick King, Business Development Manager, School of Computing \& Communication
}

Connected Health Cities (CHC) is a 20 million pound project funded by the Department of Health through the Northern Health Science Alliance. The main aim of the project is to use hospital data to improve health outcomes. The money is devolved into geographical regions that represent the areas covered by the respective Academic Health Science Networks (AHSNs) - the North West Coast, North East and North Cumbria, Connected Yorkshire and Greater Manchester. The co-ordinating centre for the initiative is located at Manchester University.

Lancaster University is in the North West Coast partnership with Liverpool University, the Innovation Agency (AHSN for the North West Coast) and AIMES grid services. The group work together on five work streams. Lancaster leads on workforce development and industry engagement. Liverpool University leads the clinical pathway work stream, AIMES the computation infrastructure, whilst the Innovation Agency leads on a work stream looking at consent. The clinical areas focused on in the North West are epilepsy, alcohol misuse and coronary obstructive pulmonary disease (COPD). The other geographical areas have chosen other clinical areas and information will be shared across the diseases. The work at Lancaster is led by Nick King (Project Lead), Jo Knight (Academic Director) and Sumi Helal (Principal Investigator).

As part of the workforce development we have an interdisciplinary cohort of $\mathrm{PhD}$ students at Lancaster University. Between them they have expertise in statistics, computing, social science and business studies. OlatunjiJohnson based in the Medical School is focused on the development and implementation of novel statistical approaches. As well as working with academic supervisors he is planning a project with Tim Gatheral, a respiratory consultant at the Royal Lancaster Infirmary. Olatunji and his supervisory team will use a locally collected dataset of emergency admissions of COPD patients to develop methods to enable real-time identification of unexplained, spatially and/or temporally localised peaks in the emergency admissions and tools to identify important risk factors. Olatunji's methods will be fed into the clinical team at Liverpool. Helena Tendedez from the School of Computing and Communication is also focused on COPD. She has carried out interviews with respiratory clinicians at Lancashire Teaching Hospital NHS Foundation Trust. The information she gained in the interviews allowed her to determine their requirements for a dashboard to provide the clinicians with the information that will help them to quickly make accurate decisions about health care requirements for individual patients. Outputs from Olatunji's project will feed into this dashboard as well as data from the Liverpool clinical team. Helena is now beginning to devise a plan for implementing the dashboard. Helena will also work on integrating hospital held data with the information that patients collect themselves using phone applications. Nicola Platt, based in the Management School, is working on the understanding of the processes behind data flow in NHS patient pathways in relation to alcohol misuse. As well as her academic supervisors she is co-supervised by Keith Bodger a consultant gastroenterologist at Aintree University Hospital in Liverpool and one of the Liverpool CHC team leads. Nicola is planning to carry out her interviews in Blackpool Teaching Hospitals NHS Foundation Trust. Victoria Neumann is a social scientist is working in the School of Computing and Communication and the aim of her project is to look at how issues of trust and privacy emerge in different consent and data sharing practices. As the newest recruit she is establishing what issues the current Connected Health City projects on consent have identified in order to shape an innovative $\mathrm{PhD}$ project. Among others Victoria will work together with Debbie Parkinson from the Innovation Agency who leads on the patient and public involvement. Debbie has been working to establish an active public involvement and engagement senate comprised of patients given an opportunity to input into the directions of the overall project. In order to ensure the clinical relevance of all the $\mathrm{PhD}$ projects the students have regular meetings with Mike Pearson a consultant with vast experience in implementing change within the NHS.

Another aspect of workforce development has been to work directly with people already in the NHS. For example Nick King has joined the Business Intelligence Skills Framework board. He has been involved in activities such as training events for directors of finance within the NHS to inform on digital subjects such as cyber security. Through the $\mathrm{CHC}$ we have also supported senior analyst training days both at Lancaster University and also at the Hartree Centre (Science and Technology Facilities Council). In February last year we hosted an event at Lancaster attended by more than 60 delegates to allow NHS Business Intelligence colleagues to share good digital practice.

At Lancaster we also take the lead on industrial engagement and hosted an Amazon Hack to develop new digital innovations to improve health care delivery. To organize the meeting we partnered with Healthier Lancashire \& South Cumbria Sustainability and Transformation Partnership, the Innovation Agency, and Amazon. One of the winning innovations was a tool incorporating IoT technology to measure fluid input and output in patients with Acute Kidney Injury.

The pathway group in Liverpool is working on hospital episode data to determine geographical locations that are hotspots for COPD, epilepsy and alcohol misuse. Further investigations of these areas can be undertaken to deliver focused interventions. This team will also be studying the pathways patients take when they are admitted and the effect on the health outcome. Such information may allow clinicians to provide more personalised treatment plans which could reduce emergency department attendances. This will improve care for the patient and reduce the cost to the NHS. AIMES grid services provide access to this data and ensure all appropriate information governance is adhered to.

While Lancaster as part of the North West Coast Group are undertaking these project the other three partnership groups are also making great strides. For example the North East and North Cumbria group are working on the Great North Care record network. In this project health professionals are teaming up to gain appropriate consent and share patient records across the different NHS organisations. One goal of $\mathrm{CHC}$ is to ensure projects that are successful in one region are reproduced in the other regions. In mid January 2018 an "all-hands" project is being held in Manchester with the express purpose of identifying the projects that can be most effectively reproduced across the regions. The central hub will then resource and enable this activity.

Correspondence to: jo.knight@lancaster.ac.uk 